\title{
Influence of the Ligand Stripping on the Transport Properties of 2 Nanoparticle-Based PbSe Nanomaterials
}

\author{
3 Doris Cadavid, ${ }^{* \dagger}$ Silvia Ortega, ${ }^{\ddagger}$ Sergio Illera, ${ }^{\S}$ Yu Liu, ${ }^{\| \odot ~ M a r i a ~ I b a ́ n ̃ e z, ~}$ \\ ${ }_{4}$ Mengyao Li, ${ }^{\ddagger}$ Antonio M. López, ${ }^{\perp}$ Germán Noriega, ${ }^{\#}$ Oscar Juan Durá, $\nabla$ M. A. López de la Torre, \\ 5 Joan Daniel Prades, ${ }^{\S}$ (i) and Andreu Cabot*, ${ }^{*}, \%$ \\ $6{ }^{\dagger}$ Departamento de Física, Universidad Nacional de Colombia, Ciudad Universitaria, 111321, Bogotá, Colombia \\ $7{ }^{\ddagger}$ Catalonia Institute for Energy Research - IREC, 08930 Sant Adrià de Besòs, Barcelona, Spain \\ $8{ }^{\S}$ Departament d'Electrònica, Universitat de Barcelona, C. Martí i Franquès 1, 08028 Barcelona, Spain \\ 9 "Institute of Science and Technology Austria, Am Campus 1, 3400 Klosterneuburg, Austria \\ $10{ }^{\perp}$ Departament d’Enginyeria Electrònica, Universitat Politècnica de Catalunya, EPSEVG, 08800 Vilanova i la Geltrú, Barcelona, Spain \\ ${ }_{11}$ \#Cidete Ingenieros S.L., Anselm Clavé 98, 08800 Vilanova i la Geltrú, Barcelona, Spain \\ $12{ }^{\nabla}$ Departamento de Física Aplicada, Universidad de Castilla-La Mancha, Avd. Camilo José Cela, Edificio Politécnico, ETSII, 13071 \\ 13 Ciudad Real, Spain \\ 14 \% ICREA, Pg. Lluís Companys 23, 08010 Barcelona, Spain
}

15 S Supporting Information

16 ABSTRACT: Nanomaterials produced from the bottom-up assembly

17 of nanocrystals may incorporate $\sim 10^{20}-10^{21} \mathrm{~cm}^{-3}$ not fully coordinated

18 surface atoms, i.e., $\sim 10^{20}-10^{21} \mathrm{~cm}^{-3}$ potential donor or acceptor states

19 that can strongly affect transport properties. Therefore, to exploit the full

20 potential of nanocrystal building blocks to produce functional

21 nanomaterials and thin films, a proper control of their surface chemistry

22 is required. Here, we analyze how the ligand stripping procedure

23 influences the charge and heat transport properties of sintered $\mathrm{PbSe}$

24 nanomaterials produced from the bottom-up assembly of colloidal $\mathrm{PbSe}$

25 nanocrystals. First, we show that the removal of the native organic

26 ligands by thermal decomposition in an inert atmosphere leaves

27 relatively large amounts of carbon at the crystal interfaces. This carbon
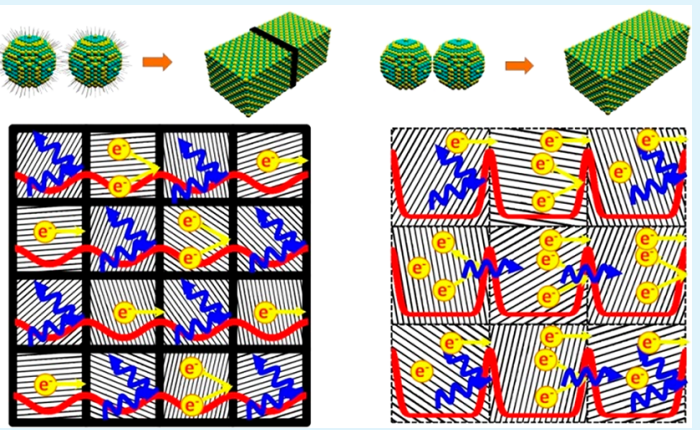

blocks crystal growth during consolidation and at the same time hampers charge and heat transport through the final nanomaterial. Second, we demonstrate that, by stripping ligands from the nanocrystal surface before consolidation, nanomaterials with larger crystal domains, lower porosity, and higher charge carrier concentrations are obtained, thus resulting in nanomaterials with higher electrical and thermal conductivities. In addition, the ligand displacement leaves the nanocrystal surface unprotected, facilitating oxidation and chalcogen evaporation. The influence of the ligand displacement on the nanomaterial charge transport properties is rationalized here using a two-band model based on the standard Boltzmann transport equation with the relaxation time approximation. Finally, we present an application of the produced functional nanomaterials by modeling, fabricating, and testing a simple PbSe-based thermoelectric device with a ring geometry.

KEYWORDS: nanoparticle, colloid, chalcogenide, thermoelectricity, ligand displacement, transport properties

\section{INTRODUCTION}

${ }_{37}$ The use of colloidal nanocrystals (NCs) with tuned size, shape, ${ }_{38}$ crystal phase, and composition as building blocks to engineer 39 nanomaterials provides unmatched control over material ${ }_{40}$ parameters at the nanometer scale and a huge design flexibility ${ }_{41}$ to optimize their functional properties. ${ }^{1-3}$ At the same time, ${ }_{42}$ bottom-up solution-processing technologies enable the produc${ }_{43}$ tion of functional materials by design in a cost-effective manner. ${ }_{44}$ These bottom-up solution-processing technologies are partic45 ularly well suited to produce thermoelectric (TE) materials and devices, which benefits from nanocrystalline materials to 46 minimize thermal conductivity. ${ }^{4-11}$

Nevertheless, to exploit the full potential of NC-based 48 bottom-up technologies to produce functional nanomaterials, 49 some limitations need to be overcome. The first limitation is the 50 presence of the electrically insulating organic surfactants used to 51

Special Issue: Thermoelectrics

Received: October 31, 2019

Accepted: December 23, 2019 
52 control NC growth, e.g., long chain alkanes and alkenes with 53 functional groups such as carboxylic and phosphonic acids, 54 thiols, or amines. These surface organic ligands block charge 55 transport, injection, and extraction among NCs and between $56 \mathrm{NCs}$ and the media, thus constraining the performance of 57 bottom-up engineered nanomaterials in all fields where charge 58 transport is involved, e.g., electronics, optoelectronics, catalysis, 59 or thermoelectricity. To remove surface organic ligands, several 60 displacement and exchange procedures, based on the use of 61 substituting shorter-surface species, have been proposed: 62 pyridine, $^{12}$ hydrazine-based metal chalcogenide com63 plexes, ${ }^{13-17}$ nitrosonium, diazonium, ${ }^{18}$ and trialkyl oxonium 64 tetrafluoroborates, ${ }^{19}$ tetrafluoroborate acids $\left(\mathrm{HBF}_{4}, \mathrm{HPF}_{6}\right){ }^{20}$ 65 tretrabutylammonium iodide, 1,2-ethanedithiol, ${ }^{21,22}$ ammo66 nium thiocianate $\left(\mathrm{NH}_{4} \mathrm{SCN}\right),{ }^{23,24} \mathrm{Na}_{2} \mathrm{~S}, \mathrm{NH}_{4} \mathrm{~S}, \mathrm{~K}_{2} \mathrm{Te}$, $67 \mathrm{~K}_{2} \mathrm{~S}^{20,24-28}$ or halide anions like $\mathrm{Cl}^{-}, \mathrm{Br}^{-}$, and $\mathrm{I}^{-},{ }^{29-32}$ among 68 others. ${ }^{19,33-37}$

69 A second limitation is the tuning of the charge carrier 70 concentration of nanomaterials obtained from the bottom-up 71 assembly of colloidal NCs. The introduction of controlled 72 amounts of external elements within the NC lattice, which is the 73 usual strategy used to dope conventional bulk or thin film 74 semiconductors, is still challenging for some compounds in $\mathrm{NC}$ 75 form. ${ }^{38-43}$ Another important limitation in this direction is the 76 control of the NC surface composition. Interfaces strongly affect 77 the electronic transport of nanomaterials through two main 78 mechanisms: (i) charge carrier scattering at the potential 79 barriers created in the grain-boundaries affects the charge carrier 80 mobility, ${ }^{44}$ and (ii) acceptor and donor states associated with 81 non-fully coordinated ions or impurities at the interfaces affect 82 the charge carrier concentration. ${ }^{45}$ Even stoichiometric and pure 83 nanomaterials obtained from the bottom-up assembly of $10 \mathrm{~nm}$ 84 NCs may incorporate $10^{20}-10^{21} \mathrm{~cm}^{-3}$ interface atoms not fully 85 coordinated, thus introducing the same amount of potential 86 donor or acceptor states. This large density of interface states 87 may translate into very large charge carrier concentrations and 88 charge transport barriers at crystal interfaces that strongly 89 influence charge transport in the nanomaterial.

90 Overcoming the two aforementioned limitations is a topic of 91 major interest for the scientific community involved in the 92 development of NC-based bottom-up technologies to produce 93 thin film, nanomaterial, and NC-based devices, such as quantum 94 dot solar cells, catalysts, or thermoelectrics. Nevertheless, most 95 of the works in this direction have focused on the study of ligand 96 exchange strategies to passivate or even introduce controlled 97 amounts of dopants in nonsintered nanoparticle arrays. In spite 98 of the extraordinary progress in this direction, nanomaterials 99 obtained by this strategy still suffer from low charge carrier 100 mobilities and relatively poor performances compared with 101 competing technologies.

102 In this work, we investigated the effect of the ligand 103 displacement (LD) procedure on the transport properties of 104 consolidated nanomaterials obtained by bottom-up strategies. 105 The influence of the ligand exchange procedure on the 106 nanomaterial transport properties is discussed in the framework 107 of a charge transport model based on the standard Boltzmann 108 transport equation ${ }^{46}$ with the relaxation time approximation and 109 developed to fit our experimental data. We used $\mathrm{PbSe}$ as the 110 model material owing to its easy synthesis with exceptional size 111 and shape control at the gram scale and its excellent TE 112 properties. In this direction, several metal chalcogenides, and 113 particularly bismuth and lead chalcogenides, ${ }^{4,7,47}$ produced 114 using a plethora of synthetic routes, including hydrothermal, ${ }^{48}$ solvothermal, ${ }^{49,50}$ sonochemical, ${ }^{51,52}$ and sol-gel, ${ }^{53}$ among 115 others, ${ }^{54-56}$ have been demonstrated to display excellent TE 116 properties. Among them, while $\mathrm{PbSe}$ has attracted less attention 117 than $\mathrm{PbTe}$ - and $\mathrm{Bi}_{2} \mathrm{Te}_{3}$-based alloys, heavily doped $\mathrm{PbSe}$ has 118 been predicted to reach TE figures of merit, ZT up to $2,{ }^{57}$ and 119 experimental $\mathrm{ZT}$ values up to 1.6 and 1.5 have been reported for 120 $\mathrm{Pb}_{1-x} \mathrm{Na}_{x} \mathrm{Se}+\mathrm{CdS}$ at $900 \mathrm{~K}$ and $\mathrm{Pb}_{0.95} \mathrm{Sb}_{0.033} \mathrm{Se}_{1-y} \mathrm{Te}$ at $823 \mathrm{~K}, 121$ respectively. ${ }^{58,59}$ Besides, $\mathrm{PbSeTe}$ alloys codoped with $\mathrm{Na}$ and 122 $\mathrm{Cu}$ and alloys of $\mathrm{PbSe}$ with $\mathrm{NaSbSe}_{2}$ have reached TE figures of 123 merit ZT up to 1.5 at $860 \mathrm{~K}^{60}$ and 1.4 near $900 \mathrm{~K},{ }^{61}$ respectively. 124

Beyond studying the influence of the ligand exchange 125 procedure on the TE properties of bottom-up engineered 126 nanomaterials, taking advantage of the versatility of solution- 127 processing technologies, we developed a TE generator with a 128 ring-shape geometry. While current commercial TE devices 129 show the conventional planar architecture and cannot adapt to 130 the huge variety of heat source/sink geometries to optimize 131 thermal energy transfer, solution-processed TE devices can be 132 fabricated with potentially any shape and size. This is here 133 demonstrated by fabricating and testing a simple TE generator 134 with a ring geometry, which can be better adapted to exhaust 135 pipes and similar thermal sources.

136

\section{EXPERIMENTAL SECTION}

Materials. Selenium pieces (99.999\%), lead acetate trihydrate 137 (99.9\%), lead nitrate (99.0\%), 1-octadecene (ODE, technical grade 138 $90 \%$ ), oleic acid (OAc, technical grade $90 \%$ ), sodium amide (95\%), 139 ammonium nitrate (98\%), anhydrous methanol, anhydrous chloro- 140 form, formamide (FA >99.5\%), and dimethylformamide (DMF 99.8\%) 141 were purchased from Aldrich. Triocthiylphosphine (TOP, 97\%) was 142 purchased from Strem Chemicals. Analytical grade ethanol, hexane, and 143 chloroform were purchased from Panreac. All chemicals were used 144 without further purification. A stock solution of TOPSe (1 M) was 145 prepared dissolving $7.89 \mathrm{~g}$ of selenium pieces in $100 \mathrm{~mL}$ of TOP. All NC 146 preparations were carried out using standard airless techniques: a 147 vacuum/dry Ar Schlenk line was used for the synthesis and an Ar-filled 148 glovebox for storing and handling oxygen- and moisture-sensitive 149 chemicals.

PbSe NCs. A modified approach to the one developed by J. J. Urban 151 et al. ${ }^{62}$ was used to synthesize PbSe NCs. In a typical procedure, $7 \mathrm{mmol} 152$ $(2.8 \mathrm{~g})$ of lead acetate trihydrate and $23 \mathrm{mmol}(6.6 \mathrm{~g})$ of OAc were 153 dissolved in $50 \mathrm{~mL}$ of ODE. The mixture was heated up to $130^{\circ} \mathrm{C}$ under 154 a vacuum for $1.5 \mathrm{~h}$. Afterward, the solution was flushed with Ar and 155 heated up to $190^{\circ} \mathrm{C}$. At this temperature, $12 \mathrm{~mL}$ of the TOPSe stock 156 solution was quickly injected. After injection, the reaction temperature 157 was maintained between 180 and $190^{\circ} \mathrm{C}$ for $10 \mathrm{~min}$. Then, the solution 158 was cooled using a water bath. PbSe NCs were precipitated and 159 redispersed several times using ethanol and hexane. Finally, PbSe NCs 160 were dispersed in chloroform and stored in the glovebox.

161

Organic Ligand Replacement. Several salts were used to displace 162 organic ligands from the $\mathrm{PbSe} \mathrm{NC}$ surface. In a typical procedure and 163 using $\mathrm{NaNH}_{2}$ as an example, PbSe NCs $(250 \mathrm{mg})$ were dispersed in 10164 $\mathrm{mL}$ of chloroform and mixed at room temperature with $5 \mathrm{~mL}$ of $\mathrm{NaNH}_{2} \quad 165$ solution $(0.01 \mathrm{M})$ in methanol. The solution was shaken for $1-2 \mathrm{~min}$ to 166 displace the organic ligands attached to the NC surface. Afterward, NCs 167 were precipitated by centrifugation and thoroughly purified using 168 chloroform/methanol to remove the remaining organic species. Finally, 169 $\mathrm{PbSe} \mathrm{NCs}$ were precipitated and dried under a vacuum or redispersed 170 in polar solvents like FA, DMF, or ethanol for further analysis. To avoid 171 the oxidation of the chalcogenide NCs, the OL replacement was 172 conducted inside the Ar-filled glovebox.

PbSe Nanomaterial. To produce PbSe bulk nanostructured 174 materials, NCs were dried under a vacuum, annealed at $450{ }^{\circ} \mathrm{C}$ for 1175 $\mathrm{h}$, and compacted at $350^{\circ} \mathrm{C}$ for $2 \mathrm{~min}$ into disk-shaped pellets of $10 \mathrm{~mm} 176$ diameter and $1 \mathrm{~mm}$ thickness under $40 \mathrm{MPa}$ of pressure. For pellet 177 preparation, we used graphite dies and a custom-made hot press 178 

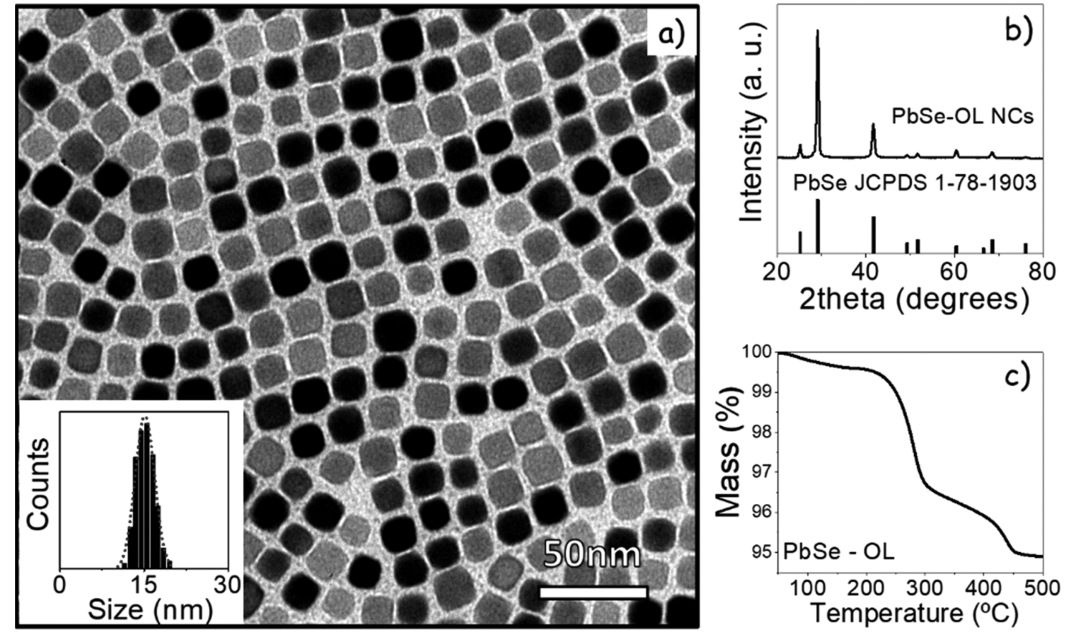

Figure 1. TEM micrograph (a) and histogram of the particle size distribution (a, inset); XRD pattern (b) and TGA profile (c) of $16 \pm 2$ nm cubeshaped PbSe-OL NCs.

179 consisting of an induction heater coupled to a hydraulic press kept 180 inside the Ar-filled glovebox.

181 Ring Thermoelectric Generator. TE rings (39 and $28 \mathrm{~mm}$, outer 182 and inner diameter, respectively, $2 \mathrm{~mm}$ thick) were built by filling two $183 \mathrm{Cu}$ concentric rings with dried $\mathrm{PbSe} \mathrm{NCs}$ followed by uniaxial hot press 184 at $350{ }^{\circ} \mathrm{C}$ and $20 \mathrm{MPa}$ for $5 \mathrm{~min}$. Rings were pressed using a custom185 made die in the hot press system previously described.

186 Structural and Chemical Characterization. X-ray power 187 diffraction (XRD) analyses were carried out on a Bruker AXS D8 188 ADVANCE X-ray diffractometer with $\mathrm{Cu} \mathrm{K} \alpha 1$ radiation $(\lambda=1.5406$ $189 \AA$ ). The size and shape of the NCs were examined by transmission 190 electron microscopy (TEM) using a ZEISS LIBRA 120, operating at $191120 \mathrm{keV}$ accelerating voltage. Scanning electron microscopy (SEM) was 192 performed using a ZEISS Auriga microscope with an energy dispersive 193 X-ray spectroscopy (EDX) detector to study the material's 194 composition. Nuclear magnetic resonance (NMR) spectra $\left({ }^{1} \mathrm{H}\right.$ 195 NMR) were acquired using a Bruker DRX 500 spectrometer equipped 196 with an $11.7 \mathrm{~T}$ magnet and an Avance III console. The spectral 197 frequency was set at $125.7 \mathrm{MHz}$ for ${ }^{13} \mathrm{C}$. ${ }^{13} \mathrm{C}$ NMR spectra were 198 referenced externally to tetramethyl silane $\left(\mathrm{SiMe}_{4}\right)$. Spectra were 199 acquired on a $2.5 \mathrm{~mm}$ MAS probe head using $20 \mathrm{kHz}$ MAS frequency at 200 room temperature. Thermal gravimetric analyses (TGA) were done 201 using PerkinElmer TGA 4000 equipment. The dried PbSe nano202 powders were heated up to $500^{\circ} \mathrm{C}$ under a nitrogen flow and a heating $203 \mathrm{ramp}$ of $5{ }^{\circ} \mathrm{C} / \mathrm{min}$. Fourier-transform infrared (FTIR) spectra were 204 acquired using an Alpha Bruker FTIR spectrometer with the platinum 205 attenuated total reflectance (ATR) single reflection module, and FTIR 206 data were typically averaged over 64 scans.

207 Thermoelectric Characterization. Seebeck coefficients and 208 electrical resistivities were measured simultaneously using a Linseis 209 LSR 3 system under a helium atmosphere. The Seebeck coefficient was 210 obtained using a static DC method, and the electrical resistivity was 211 measured by means of a standard four-probe technique in the 212 temperature range from 300 to $600 \mathrm{~K}$. Thermal conductivity data 213 were obtained from flash diffusivity measurements (Linseis LFA 1000) 214 as a function of temperature. The thermal conductivity was calculated 215 as $\kappa=D C_{p} d$, where $D$ is the thermal diffusivity, $C_{p}$ is the heat capacity, 216 and $d$ is the density. $C_{p}$ was approximated by the formula $0.171+(2.65$ $\left.217 \times 10^{-5}\right) T$, where $T$ is the absolute temperature. ${ }^{63-65}$ At $T>T_{D}$ (where $218 T_{\mathrm{D}}$ is the Debye temperature), this equation is consistent with the 219 higher $C_{p}$ values reported for nanostructured materials, as compared 220 with values obtained by the Dulong-Petit approximation. ${ }^{66}$ These 221 alterations of $C_{p}$ values are caused probably by the high surface to 222 volume ratio in nanostructured materials. Room temperature measure223 ments of the Hall charge carrier concentration and mobility were 224 performed in a four-probe configuration using an electromagnet that 225 generated a magnetic field up to $1.2 \mathrm{~T}$. Current was passed through the sample while the field was varied between 0.1 and $1 \mathrm{~T}$ in increments of 226 $0.1 \mathrm{~T}$ with an inversion of the field to eliminate voltage probe 227 misalignment effects.

Device Test. Ring TE generators were tested in a custom-made test 229 bench. The inner part of the rings was heated using a cylindrical metal 230 block containing a heating cartridge, while the outer part was 231 maintained cold by means of commercial TE modules attached to a 232 large stainless steel plate. Starting from a $10{ }^{\circ} \mathrm{C}$ thermal gradient at 233 room temperature, temperature was increased gradually until reaching 234 $250{ }^{\circ} \mathrm{C}$ at the hot side, while the cold side was kept at $60{ }^{\circ} \mathrm{C}$, thus 235 generating a maximum temperature gradient of $190^{\circ} \mathrm{C}$. Temperature 236 was monitored with two thermocouples attached to the cold and hot 237 sides of the ring, respectively, and the open circuit voltage generated 238 between the two $\mathrm{Cu}$ electrodes was measured using a Keithley 2400239 multimeter.

\section{RESULTS AND DISCUSSION}

$\mathrm{PbSe} \mathrm{NCs}$ were synthesized by reacting lead acetate with 241 TOPSe at $190^{\circ} \mathrm{C}$. The presence of OAc in the reacting mixture 242 was essential to limit the $\mathrm{PbSe} \mathrm{NC}$ growth during their synthesis 243 and to colloidally stabilize them. As-produced PbSe NCs, 244 henceforth referred to as $\mathrm{PbSe}-\mathrm{OL}$, had an average size of $16 \pm 2245 \mathrm{fl}$ $\mathrm{nm}$ with an $\sim 10 \%$ size dispersion, cubic shape (Figure 1a) with $246 \mathrm{fl}$ [100] facets, and face centered cubic crystallographic phase (fcc, 247 JCPDS 1-78-1903, Figure 1b).

248

A total of $6 \%$ of the total mass of the PbSe-OL NCs obtained 249 after purification with three precipitation and redispersion steps 250 was assigned to surface ligands according to TGA (Figure 1c). 251 The solid state ${ }^{13} \mathrm{C}$ NMR spectrum of PbSe-OL NCs after 252 purification displayed the characteristic $\mathrm{C}=\mathrm{C}$ peak and the 253 $\mathrm{COOH}$ band associated with OAc. We therefore concluded 254 $\mathrm{OAc}$ to be the only ligand present at the PbSe-OL NC surface. 255 Taking into account the organic amount measured by TG, a 256 ligand surface concentration of $\sim 1.4 \mathrm{~nm}^{-2}$ was estimated. $\quad 257$

The presence of organic ligands at the NC surface was 258 anticipated to strongly limit the performance of any NC-based 259 application that involves charge transport/transfer between/ 260 from/to NCs, e.g., photovoltaics, electroluminescent light 261 emitting diodes, thermoelectrics, and catalysis. ${ }^{67-71}$ To take 262 full advantage of the convenient processability of colloidal NCs, 263 organic ligands can be chemically or thermally removed after 264 their deposition/assembly. For dense nanomaterials and 265 relatively thick films, chemical processes are not effective 266 because they may not reach the full NC surface. Thus, thermal 267 
268 decomposition becomes the only viable procedure. Alter269 natively, native organic ligands can be exchanged in solution, 270 before NC deposition/assembly, by smaller organic or inorganic 271 species able to maintain the NCs colloidally stable, but having a 272 minor effect on the nanomaterial transport properties. There are 273 a myriad of potential ligands that may provide the aforemen274 tioned conditions, from metal free anionic ligands $\left(\mathrm{OH}^{-}, \mathrm{NH}_{2}^{-}\right)$ 275 that provide electrostatic stabilization of the NCs to metal 276 chalcogenide complexes MCC obtained using hydrazine ${ }^{33}$ to 277 short thiols, e.g., 1,2-ethanedithiol EDT, frequently used in the 278 photovoltaics field. ${ }^{21,22}$ Among the potential candidates to 279 displace organic ligands from the PbSe-OL NC surface, 280 hydrazine was discarded due to its toxicity and dangerous 281 manipulation, and sulfur-based compounds were not considered 282 due to their potential to sulfurize the selenide NCs. Thus, other 283 alternative inorganic salts were tested.

284 To test different salts to displace OAc, PbSe-OL NCs 285 dispersed in chloroform were mixed with a $0.01 \mathrm{M}$ solution of 286 the salt in methanol. The resulting mixture was vigorously 287 shaken at room temperature for a few minutes. During this 288 process, OAc was stripped from the $\mathrm{PbSe}-\mathrm{OL} \mathrm{NC}$ surface and 289 the resulting charge-stabilized $\mathrm{PbSe} \mathrm{NCs}$ moved to the methanol 290 phase. The obtained PbSe NCs, henceforth referred to as PbSe$291 \mathrm{LD}$, were thoroughly washed with chloroform to remove the 292 remaining organic species and finally precipitated and dried 293 under a vacuum. All of these processes were carried out inside an 294 Ar-filled glovebox to avoid oxidation. PbSe-LD NCs preserved 295 the shape and size distributions of the original PbSe-OL NCs 296 (Figure S1) and were dispersible in polar solvents like FA, DMF, 297 or alcohols for a limited amount of time.

298 In order to characterize the efficiency of the ligand 299 displacement, we used FTIR and NMR spectroscopy. The 300 FTIR spectra for PbSe-LD show that the peaks corresponding to 301 organic ligands are barely visible after the LD (Figure S2), 302 indicating a successful treatment. Solid state ${ }^{13} \mathrm{C}$ NMR analysis 303 of the $\mathrm{PbSe} \mathrm{NCs}$ treated with $\mathrm{NaNH}_{2}$, henceforth referred to as $304 \mathrm{PbSe} \mathrm{NaNH}_{2}$, showed the OAc fingerprints to vanish after the 305 ligand displacement process (Figure 2). While a previous work

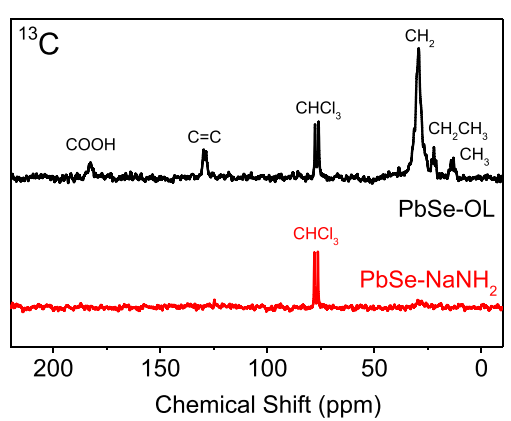

Figure 2. Solid state ${ }^{13} \mathrm{C}$ NMR spectra of $\mathrm{PbSe}-\mathrm{OL}$ (black) and $\mathrm{PbSe}-$ $\mathrm{NaNH}_{2}$ (red) powders dried from a $\mathrm{CHCl}_{3}$ solution. The peaks displayed in the ${ }^{13} \mathrm{C}$ NMR spectra of $\mathrm{PbSe}-\mathrm{OL}$ are consistent with the OAc fingerprint. The ${ }^{13} \mathrm{C}$ NMR spectra of $\mathrm{PbSe}-\mathrm{NaNH}_{2}$ demonstrated the removal of OAc during ligand displacement.

306 identified $\mathrm{NH}_{2}$ as the species remaining at the surface of CdSe $307 \mathrm{NCs}^{20}$ no evidence of $\mathrm{NH}_{2}{ }^{-}$fingerprints in the ${ }^{1} \mathrm{H} \mathrm{NMR}$ 308 spectrum of $\mathrm{PbSe}-\mathrm{NaNH}_{2} \mathrm{NCs}$ could be found. We associated 309 this fact to the facile decomposition of the amide group. It 310 should also be mentioned in this regard that the ligand 311 displacement procedure was carried out in a glovebox, but for 312 NMR characterization, NCs were exposed to air, which may have modified their surface chemistry, especially taking into 313 account the high reactivity of the amide group.

To produce nanostructured $\mathrm{PbSe}, \sim 1 \mathrm{~g}$ of $\mathrm{PbSe} \mathrm{NCs}$ was 315 thoroughly cleaned, dried, and annealed at $450{ }^{\circ} \mathrm{C}$ for $1 \mathrm{~h}$ in 316 argon. The annealed material was afterward compacted at 350317 ${ }^{\circ} \mathrm{C}$ under $40 \mathrm{MPa}$ of pressure using a hot press. The relative 318 densities of the final PbSe-OL pellets were around $85 \%$. Those 319 of the PbSe-LD pellets were systematically higher, above $90 \% .320$ The annealing of PbSe-OL under an inert atmosphere caused 321 the thermal decomposition of the surface OLs. This 322 decomposition left a relatively large amount of carbon, which 323 was quantified by elemental analysis at around $2 \%$. On the other 324 hand, the residual carbon within the final PbSe-LD nanocrystal- 325 line powders was within the limit of detection of our system, 326 $\sim 0.1 \%$. EDX and ICP analysis of $\mathrm{PbSe}-\mathrm{NaNH}_{2}$ showed no 327 evidence of the presence of $\mathrm{Na}$, pointing out the inefficiency of 328 doping PbSe with $\mathrm{Na}$ by this procedure. ${ }^{63,65,72,73} 329$

During the thermal treatments, the average PbSe-OL crystal 330 size increased from the initial $\sim 16 \mathrm{~nm}$ to $\sim 20 \mathrm{~nm}$ (Figure 3). $331 \mathrm{f} 3$ The average crystal domain size of the thermal treated PbSe-LD 332 materials was systematically larger. For the $\mathrm{PbSe}-\mathrm{NaNH}_{2} 333$ sample, the crystal size domain increased from 16 to 25-30 334 $\mathrm{nm}$ with the two thermal treatments (Figure 3 ). These results 335 were confirmed by analyzing the XRD patterns using the 336 Scherrer equation, which showed that the grain size of the 337 compacted material increases up to 1.5 times with respect to the 338 NCs when the NCs were processed without LD. The size 339 increased up to 2.5 times when the NCs were processed after LD 340 (Table S1). We associate the smaller size of the crystal domains 341 obtained from the thermal treatment of PbSe-OL NCs with the 342 creation of a carbon layer that hindered crystal growth. 343

Figure 4 displays the electrical conductivity $(\sigma)$, Seebeck $344 \mathrm{f4}$ coefficient $(S)$, thermal conductivity $(\kappa)$, power factor $(\mathrm{PF})$, and 345 TE figure of merit $(\mathrm{ZT})$ of consolidated $\mathrm{PbSe}-\mathrm{OL}$ and $\mathrm{PbSe}-\mathrm{LD} 346$ samples. Electrical conductivities of the $\mathrm{PbSe}-\mathrm{OL}$ samples were 347 relatively low, below $10^{4} \mathrm{~S} \mathrm{~m}^{-1}$. The temperature dependence of 348 the electrical conductivities displayed a step increase in the range 349 from 400 to $500 \mathrm{~K}$. This step was accompanied by a parallel step 350 change of the Seebeck coefficient, which included a sign 351 inversion at around $400 \mathrm{~K}$, from positive to negative values. 352 These step changes and the sign inversion of the Seebeck 353 coefficient suggested the thermal activation of a large 354 concentration of negatively charged carriers above $400 \mathrm{~K} . \quad 355$

The stripping of the organic ligands before consolidation 356 resulted in a strong increase, up to 2 orders of magnitude, of the 357 electrical conductivity when compared with $\mathrm{PbSe}-\mathrm{OL}$ samples 358 (Figure 4a). For $\mathrm{PbSe}^{-\mathrm{NaNH}_{2}}$, a slight decrease of the electrical 359 conductivity with temperature was observed, consistent with a 360 degenerated semiconductor behavior. Besides, negative Seebeck 361 coefficients with monotonically increasing absolute values were 362 measured for all of the PbSe-LD materials in the entire 363 temperature range tested (Figure 4b). The maximum PF 364 (Figure $4 \mathrm{c}$ ) was around $1.1 \mathrm{~mW} \mathrm{~m}^{-1} \mathrm{~K}^{-2}$. This value was close to 365 the one reported by Ibañez et al., for $\mathrm{PbTe}_{x} \mathrm{Se}_{y} \mathrm{~S}_{1-x-y} 366$ nanocomposites with $\mathrm{HCl}$-mediated ligand displacement, ${ }^{29} 367$ but it was higher than the maximum values previously reported 368 for ligand-free $\mathrm{PbSe} \mathrm{NCs}{ }^{74}$ and mixed $\mathrm{PbTe} / \mathrm{PbSe} \mathrm{NCs} .^{75} \quad 369$

The absolute values of the Seebeck coefficient were lower for 370 $\mathrm{PbSe}-\mathrm{LD}$ than for $\mathrm{PbSe}-\mathrm{OL}$, which denoted higher charge 371 carrier concentrations in the former, in concordance with Hall 372 charge carrier concentrations measured at room temperature: $n_{\mathrm{H}} 373$ $=2.2 \times 10^{19} \mathrm{~cm}^{-3}$ for PbSe-LD and $n_{\mathrm{H}}=5 \times 10^{15} \mathrm{~cm}^{-3}$ for PbSe- 374 OL (Table S2). All of the inorganic ligands tested here resulted 375 

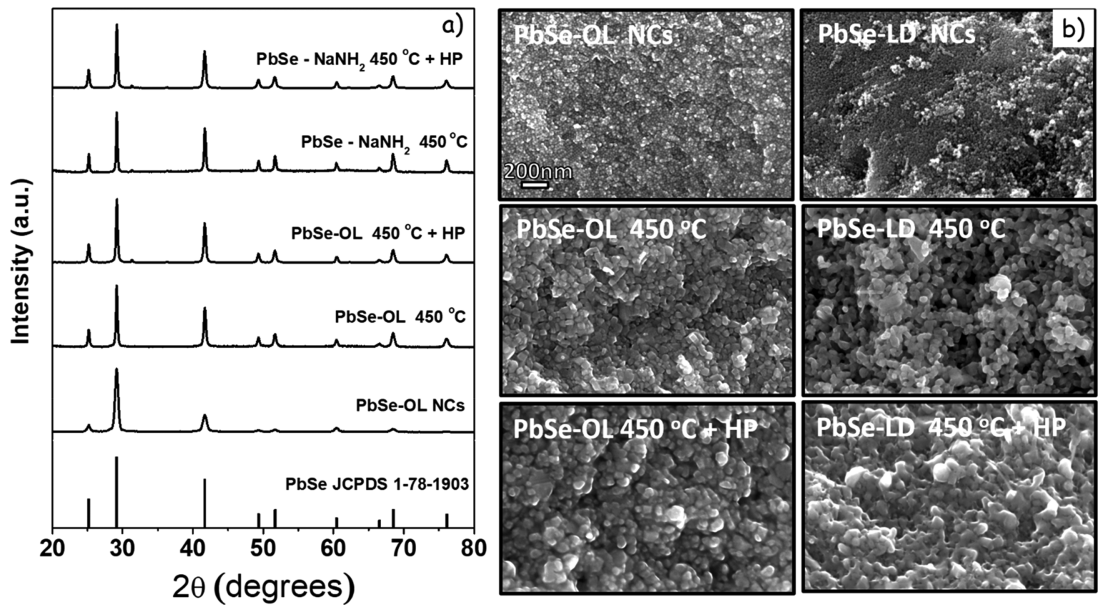

Figure 3. XRD patterns (a) and SEM images (b) of PbSe-OL and PbSe-LD with no thermal treatment (NCs), after heat treatment at $450{ }^{\circ} \mathrm{C}\left(450{ }^{\circ} \mathrm{C}\right)$ and after additional hot press at $350^{\circ} \mathrm{C}$ and $4 \mathrm{MPa}$ for $2 \min \left(450^{\circ} \mathrm{C}+\mathrm{HP}\right)$.
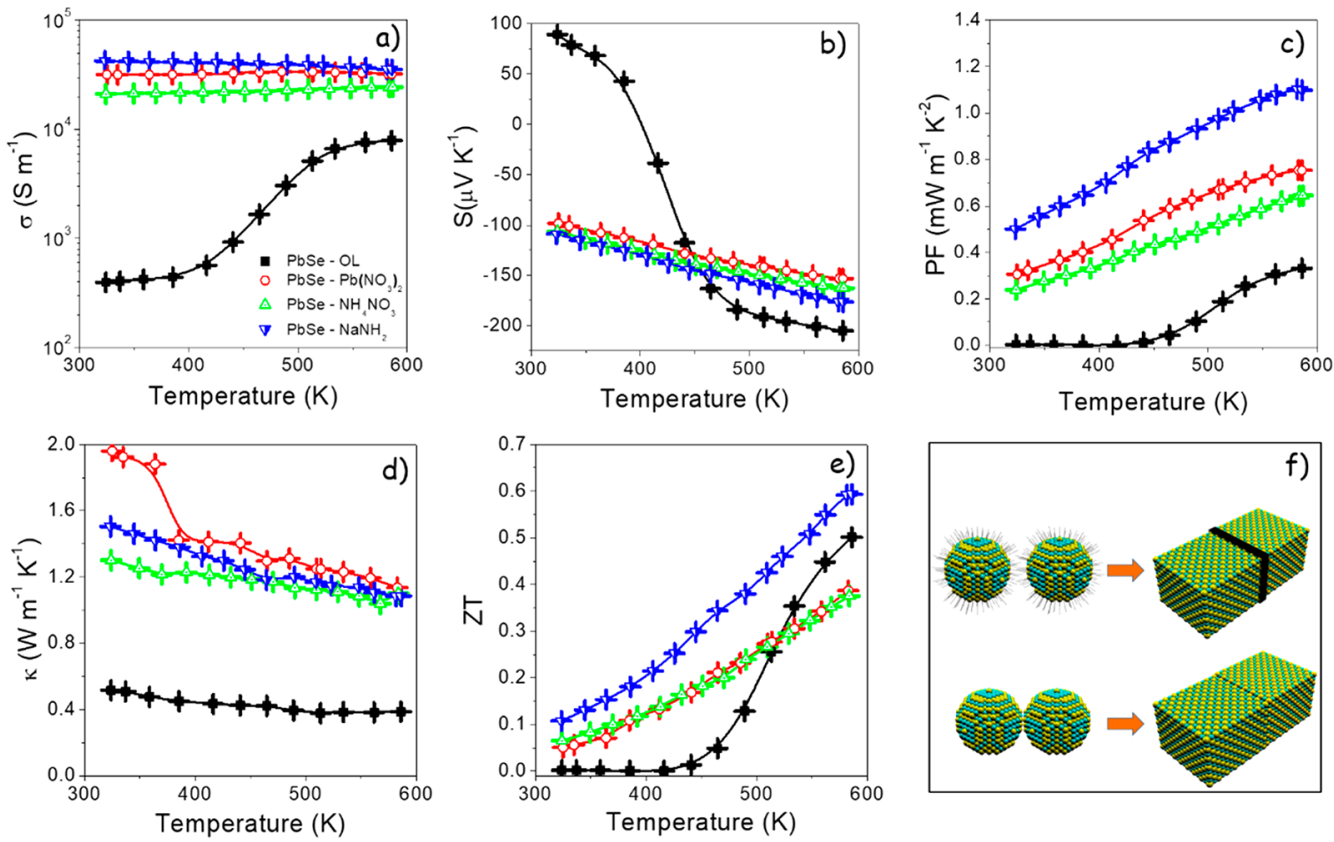

Figure 4. (a-e) Electrical conductivity, $\sigma(\mathrm{a})$, Seebeck coefficient, $S(\mathrm{~b})$, power factor, PF (c), thermal conductivity, $\kappa$ (d), and thermoelectric figure of merit, ZT (e) of nanocrystalline PbSe obtained from the bottom-up assembly of PbSe-OL and PbSe-LD NCs. LD was carried out using different capping agents, as labeled in the first panel (a). The same color was used for all panels. (f) Scheme of the nanomaterial obtained with and without ligand displacement.

376 in a similar temperature dependence of the transport properties. 377 However, $\mathrm{NaNH}_{2}$ was the salt resulting in a larger electrical 378 conductivity increase, which we associated with the easy 379 decomposition of the amide group during the NC consolidation. 380 We believe that the large differences in the temperature 381 dependence of the electrical conductivity and Seebeck 382 coefficient obtained between $\mathrm{PbSe}-\mathrm{OL}$ and $\mathrm{PbSe}-\mathrm{LD}$ samples 383 were in large part related to significant variations of the charge 384 carrier concentration. This variation of the charge carrier 385 concentration was related to changes in the material 386 composition associated with the different organic ligand removal 387 strategies. Initial colloidal NCs were uncharged because OAc 388 molecules compensated for the surface dangling bonds. The 389 thermal decomposition of the $\mathrm{OAc}$ bond to $\mathrm{Pb}$ ions at the $\mathrm{PbSe}$ 390 surface left oxygen ions behind, that compensated surface charge 391 or trapped free charges. ${ }^{29,76}$ We experimentally observe that the potential $10^{20}-10^{21}$ dangling bonds in $\mathrm{PbSe}-\mathrm{OL}$ translated in 392 just $10^{16}$ charge carriers, as measured by Hall. On the other hand, 393 the stripping of the OAc molecules with $\mathrm{NaNH}_{2}$ resulted in NCs 394 with $\mathrm{Pb}$-rich surfaces and an overall off-stoichiometric 395 composition. Thus, high concentrations of free electrons were 396 measured. The Hall charge carrier concentration at room 397 temperature was $n_{\mathrm{e}}=2 \times 10^{19} \mathrm{~cm}^{-3}$ for $\mathrm{PbSe}_{-\mathrm{NaNH}_{2}}$. In 398 addition, during the thermal treatments, some amount of Se 399 could leave the NCs, intensifying the stoichiometry unbalance. 400 This effect may be insignificant in $\mathrm{PbSe}-\mathrm{OL}$ samples, as the 401 carbon left after the organic thermal decomposition may better 402 protect the material from the chalcogen loss.

403

To further understand the electrical conductivity and Seebeck 404 coefficient evolution with temperature, a charge transport model 405 based on the standard Boltzmann transport equation ${ }^{46}$ with the 406 relaxation time approximation was used (see the Supporting 407 

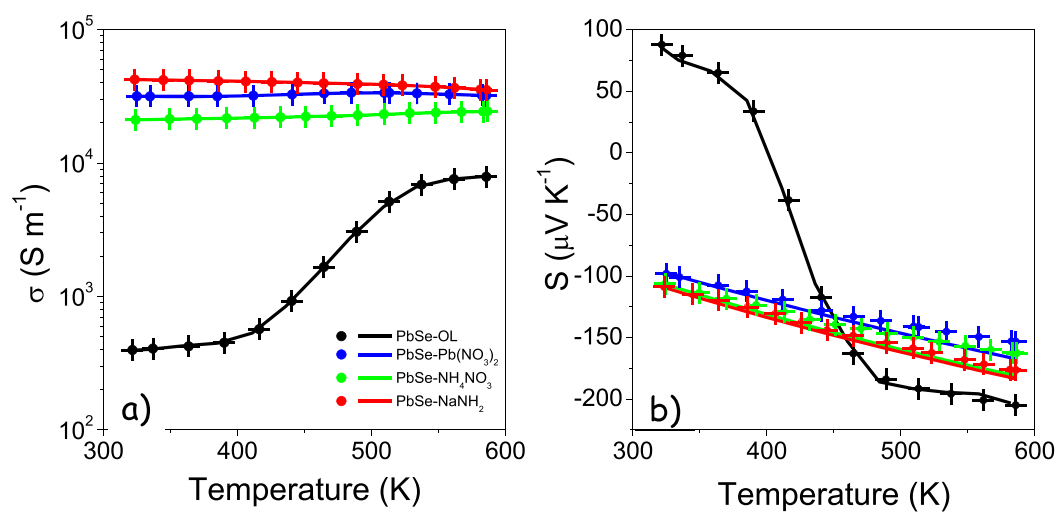

Figure 5. (a) Electrical conductivity and (b) Seebeck coefficient as a function of temperature. Symbols display the obtained experimental values, and the plotted continuous line was calculated from the two-band model.

408 Information). The electrical conductivity and the Seebeck 409 coefficient of the $\mathrm{PbSe}-\mathrm{OL}$ and $\mathrm{PbSe}-\mathrm{LD}$ nanomaterials were 410 fitted using this two-band model in the temperature range from 411300 to $600 \mathrm{~K}$. The parameters that describe the phonon 412 scattering processes were fixed for all materials and were not 413 allowed to change with temperature (Table S2). Using the 414 material parameters of Table S2, it was not possible to reproduce 415 the experimental values. Thus, we included an interface 416 scattering as an infinite series of potential barriers to reflect 417 the material discontinuity between NCs, reducing the electrical 418 conductivity of the sample with respect to the bulk case. 419 Interface scattering parameters were adjusted for each sample to 420 simultaneously fit both electrical conductivity and Seebeck 421 coefficient tendencies (Figure 5).

422 Taking into account the interface scattering, the two-band 423 model successfully reproduced the complex temperature 424 evolution of the electrical conductivity and Seebeck coefficient 425 of $\mathrm{PbSe}-\mathrm{OL}$ (Figure 5). The electrical conductivity increase with 426 temperature and the complex Seebeck trend reflected an 427 intrinsic semiconductor behavior. The sign change in the 428 temperature evolution of the Seebeck coefficient and the 429 different slopes of the electrical conductivity curves pointed at 430 two different regimes in which each type of charge carrier 431 dominated. In the low temperature region $(300 \mathrm{~K}<T<420 \mathrm{~K})$, 432 the electrical conductivity remained approximately constant and 433 the Seebeck coefficient was positive; thus, holes dominated the 434 charge transport in the material. In the intermediate temperature 435 region $(420 \mathrm{~K}<T<520 \mathrm{~K})$, as temperature increased, the 436 Seebeck coefficient rapidly decreased to zero, reflecting a similar 437 electron and hole contribution. In the highest temperature range $438(520 \mathrm{~K}<T<600 \mathrm{~K})$, electrons dominated the electrical 439 conductivity, as reflected in the negative Seebeck coefficients 440 experimentally measured.

441 For $\mathrm{PbSe}-\mathrm{OL}$, we used the parameters of Table S2 to describe 442 the phonon scattering. We additionally included the interface 443 scattering in the scattering rates of both electrons and holes. The 444 height and the width of the barrier were used as fitting 445 parameters, whereas the grain size was set to $L=25 \mathrm{~nm}$, as we 446 inferred from XRD measurements. The heights of the barrier for 447 each carrier were assumed independent from each other but 448 dependent on temperature. The initial hole concentration that 449 best fitted the experimental results was $p=5 \times 10^{15} \mathrm{~cm}^{-3}$, and 450 the best energy barrier was $0.1 \mathrm{eV}$.

451 On the other hand, for the $\mathrm{PbSe}-\mathrm{NaNH}_{2}$ sample, the increase 452 of the Seebeck coefficient and the decrease of the electrical 453 conductivity with temperature denoted that the material was completely degenerated in the whole temperature range. Thus, 454 this trend could be well explained using only the majority 455 carriers, electrons in this case. We assumed that the electron 456 concentration remained constant in the whole temperature 457 range at $n=\mathrm{Nd}$ (where $\mathrm{Nd}$ is the doping concentration with all 458 of the impurities being ionized), while the scattering times 459 decreased with temperature. Taking into account the Hall 460 results, the doping concentration was set to $\mathrm{Nd}=2 \times 10^{19} \mathrm{~cm}^{-3} \cdot 461$ This high charge carrier concentration was associated with a 462 stoichiometry unbalance produced during the OAc removal and 463 potentially from a small selenium evaporation during thermal 464 treatments as explained above. We used the same parameters for 465 $\mathrm{PbSe}-\mathrm{OL}$ and $\mathrm{PbSe}-\mathrm{LD}$ to account for the phonon scatterings. 466 To describe the interface scattering, we used a fixed barrier width 467 of $w=3 \mathrm{~nm}$ and a grain size of $L=30 \mathrm{~nm}$, as given from XRD 468 results. The barrier height was used as a fitting parameter. With 469 the introduction of interface scattering, the experimental 470 conductivity and Seebeck coefficients were well reproduced by 471 the two-band model when considering a lineal temperature 472 dependence of the chemical potential $\mu=0.2879+3.4164 \times 473$ $10^{-4} \mathrm{~T}$, measured from the top of the valence band. This result 474 was consistent with a completely degenerated semiconductor, 475 since the chemical potential was always inside the conduction 476 band in the whole temperature range. Concerning the interface 477 scattering, the barrier height was temperature dependent, 478 following a lineal trend described by $E_{\mathrm{b}}=0.26699-2.2495 \times 479$ $10^{-4} \mathrm{~T}$, which resulted in effective barriers slightly above those of 480 $\mathrm{PbSe}-\mathrm{OL}$ in the temperature range tested.

Figure $4 \mathrm{~d}$ displays the thermal conductivity $(\kappa)$ of the 482 different PbSe materials in the temperature range $300-600 \mathrm{~K} .483$ Thermal conductivities monotonically decreased with temper- 484 ature for all nanomaterials. $\mathrm{PbSe}-\mathrm{OL}$ was characterized by up to 485 3 -fold lower thermal conductivities than PbSe-LD in the 486 measured temperature range. These experimental results proved 487 that the products from the decomposition of the OLs in the 488 material not only blocked crystal domain growth, charge carrier 489 transport, and possibly Se evaporation but also affected the 490 phonon propagation. The lower thermal conductivities obtained 491 for $\mathrm{PbSe}$-OL had their origin on the carbon present in between 492 $\mathrm{NCs}$, the slightly larger interface density of $\mathrm{PbSe}-\mathrm{OL}$ related 493 with the smaller size of the crystal domains, and the higher 494 porosity of the $\mathrm{PbSe}-\mathrm{OL}$ when compared to $\mathrm{PbSe}-\mathrm{LD}$. 495

Finally, the TE figure of merit, $\mathrm{ZT}=T S^{2} \sigma / \kappa$, increased with 496 temperature for all materials, reaching values up to 0.5 for $\mathrm{PbSe}-497$ $\mathrm{OL}$ and 0.6 for $\mathrm{PbSe}^{-\mathrm{NaNH}_{2}}$ (Figure 4e). These values were 498 significantly higher than those of undoped bulk $\mathrm{PbSe}$ and 499 
500 comparable with those obtained from bulk PbSe doped with $\mathrm{Ag}$ 501 or $\mathrm{Na}$ at $600 \mathrm{~K} .^{63,72}$ By measuring the same sample for several 502 consecutive temperature cycles, we observed the transport 503 properties of the $\mathrm{PbSe}$ nanomaterials to be stable in the 504 measured temperature range (Figure S3).

505 To further demonstrate the suitability of this strategy to 506 produce functional materials and devices, $\mathrm{PbSe}-\mathrm{NaNH}_{2}$ nano507 materials were employed to build ring-shaped TE generators. ${ }^{6,77}$ 508 While most of the commercially available TE devices are plate509 shaped, TE devices build on a ring configuration are very 510 convenient and appealing when heat to be harvested or delivered 511 diffuses in a radial direction, e.g., in pipes transporting a hot 512 fluid. ${ }^{1}$ The assembly of several $\mathrm{n}$ - and $\mathrm{p}$-type TE rings connected 513 thermally in parallel and electrically in series would result in a 514 cylindrical-tube TE generator with improved thermal contact 515 between the hot surface and the device, maximizing the thermal 516 power collected or delivered.

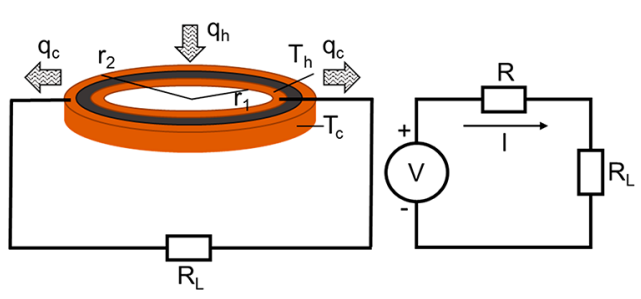

Figure 6. Basic electrical model of a ring TE generator.

517 In Figure 6, the basic electrical model of the ring TE generator 518 is shown, where electrical power generated by a TE device can be 519 generally expressed as ${ }^{78}$

$520 \quad P_{\text {gen }}=q_{\mathrm{h}}-q_{\mathrm{c}}$

521 where $q_{\mathrm{h}}$ and $q_{\mathrm{c}}$ are the incoming and outgoing thermal power in 522 the hot and cold side of the TE generator, respectively. 523 Assuming mean values for $S, \sigma$, and $\kappa$, considering $I$ as the 524 generated electrical current that flows through the closed loop 525 circuit, $R$ as the electrical resistance of solely the TE material, 526 and $T_{\mathrm{h}}$ and $T_{\mathrm{c}}$ as the temperatures in the hot and cold sides, 527 respectively,

528

$$
q_{\mathrm{h}}=\operatorname{SIT}_{\mathrm{h}}-\frac{1}{2} I^{2} R+\kappa \Delta T
$$

$$
q_{c}=S I T_{c}+\frac{1}{2} I^{2} R+\kappa \Delta T
$$

In the load resistance, the electrical power can be redefined as 530

$$
P_{\text {gen }}=S I \Delta \mathrm{T}-I^{2} R
$$

Therefore, the maximum electrical power $P_{\max }$ is obtained when 532 the load $R_{\mathrm{L}}$ has the same value as the internal resistance $R \quad 533$

$$
\frac{\partial P_{\text {gen }}}{\partial I}=0=S \Delta T-2 I R \rightarrow I_{\max }=\frac{S \Delta T}{2 R}
$$

considering that the resistivity of a single ring is found $\mathrm{by}^{79}$ 535

$$
R=\frac{1}{2 \pi t \sigma} \int_{r_{1}}^{r_{2}} \frac{1}{r} \mathrm{~d} r=\frac{1}{2 \pi t \sigma} \ln \frac{r_{2}}{r_{1}}
$$

where $t$ is the thickness of the TE material. Then, the maximum 537 electrical power of one TE ring generator can be expressed as a 538 combination of material parameters:

$$
P_{\max }=\frac{(S \Delta T)^{2}}{4 R}=\frac{\pi t \sigma(S \Delta T)^{2}}{2 \ln \frac{r_{2}}{r_{1}}}
$$

A single n-type ring, $38 \mathrm{~mm}$ external diameter and $29 \mathrm{~mm} 541$ internal diameter, was fabricated by filling with dried surface- 542 engineered $\mathrm{PbSe} \mathrm{NCs}$ the $1.5 \mathrm{~mm}$ empty space between two 543 concentric $\mathrm{Cu}$ rings and hot pressing the material at $350{ }^{\circ} \mathrm{C} 544$ (Figure 7c). The ring was tested in a custom-made test bench. $545 \mathrm{f7}$ To create a radial temperature gradient, the ring was fitted 546 between two electrically isolated metal blocks. The inner 547 cylindrical block incorporated a heat cartridge and provided heat 548 to the internal part of the ring. The metal block around the ring 549 was kept at a relatively low temperature by means of commercial 550 TE modules attached to it. The open circuit voltage was 551 measured while heating the inner metal ring up to $530 \mathrm{~K}$ (Figure 552 $7 \mathrm{a}$ ). The measured voltage generated, around $30 \mathrm{mV}$ at $190 \mathrm{~K} 553$ thermal gradient, was in good agreement with measurements of 554 the Seebeck coefficient of the material (Figure 4b). The ring 555 electrical resistance was measured at $1.75 \mathrm{~m} \Omega$, which translated 556 into a maximum power output of around $500 \mathrm{~mW}$ (Figure $7 \mathrm{~b}$ ) at 557 the maximum temperature difference reached at the test bench 558 $(190 \mathrm{~K})$. Values obtained here were higher than those previously 559 reported with a similar geometry ${ }^{6}$ due to the larger size of the 560 present ring, the higher Seebeck coefficient of the materials used 561 here, and the higher temperature difference tested in the present 562
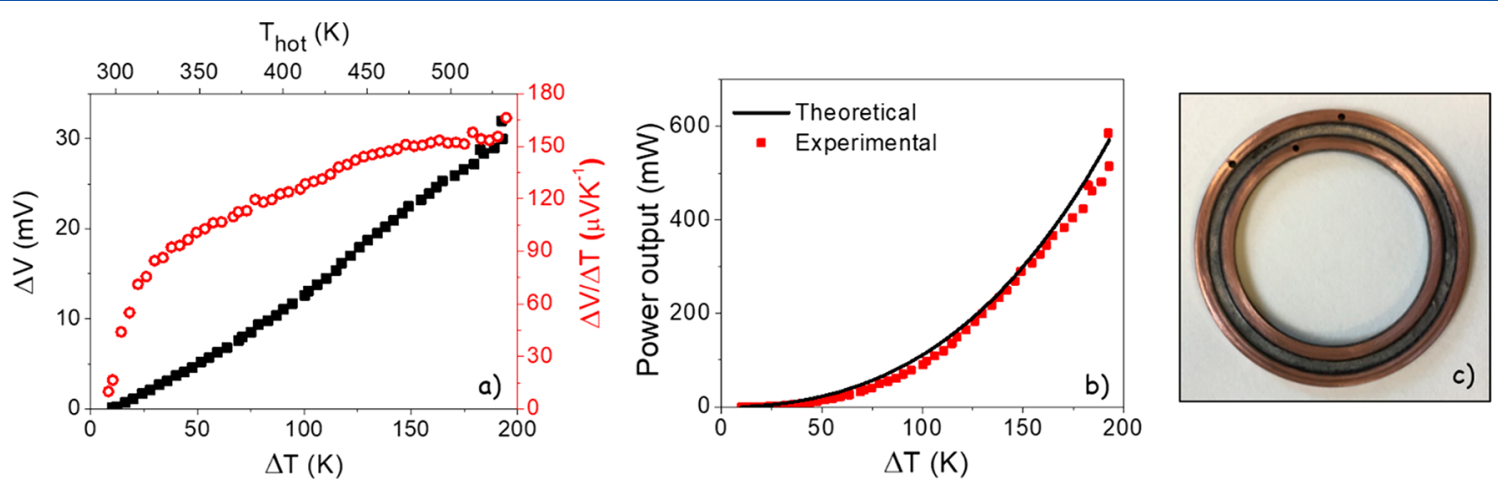

Figure 7. (a) Open circuit voltage (black squares) obtained from a single PbSe ring as a function of the temperature gradient generated by increasing the temperature at the hot side (internal part of the ring) and $S$ (red open circles). (b) Power output of the PbSe ring as a function of the thermal gradient. Red squares represent the experimental data collection and the solid black line the theoretical calculated values taking into account experimental values for $S$ and $\sigma$ from the PbSe-LD bulk nanomaterial. (c) Image of the TE device with ring geometry. 
563 work. Experimental values were consistent with results from the 564 electrical model, although the latter were slightly higher due to 565 the omission of contact resistance in the model. Nevertheless, 566 these results indicated the high potential of not only the ring 567 configuration but also the solution-processed and bottom-up 568 engineered TE materials and shape-adaptable devices.

\section{CONCLUSIONS}

569 In summary, PbSe nanomaterials were produced from the 570 bottom-up assembly of PbSe colloidal NCs synthesized in the 571 presence of OAc. The effect of two organic ligand stripping 572 strategies was analyzed. On one hand, the organic removal by 573 thermal decomposition resulted in a reduced crystal growth and 574 the presence of relatively large amounts of carbon in the final 575 material. Both lead to reduced electrical and thermal 576 conductivities due to enhanced charge carrier and phonon 577 scattering. Additionally, the relatively low electrical conductiv578 ities measured for PbSe nanomaterials obtained after the 579 thermal decomposition of the organic ligands were associated 580 with a moderate charge carrier concentration. On the other 581 hand, the OAc displacement by metal salts had associated the 582 introduction of a relatively large charge carrier density through 583 the NC surface. This charge increased the interface energy 584 barriers and the concentration of free charge carriers, resulting in 585 a semiconductor degeneration. As a result, much higher 586 electrical conductivities but also lower Seebeck coefficients 587 were obtained for $\mathrm{PbSe}$ nanomaterials obtained from the 588 consolidation of ligand-displaced PbSe NCs. Additionally, due 589 to the higher density, the larger crystal domains, and the reduced 590 phonon scattering efficiency of $\mathrm{PbSe}-\mathrm{LD}$ when compared with $591 \mathrm{PbSe}-\mathrm{OL}$ and also due to an increase of the electronic 592 contribution, the thermal conductivity of these materials was 593 relatively larger. Finally, a ring-shaped TE device was fabricated 594 and tested, delivering a maximum power output of around 500 $595 \mathrm{~mW}$ with $190 \mathrm{~K}$ temperature difference.

\section{ASSOCIATED CONTENT}

\section{S Supporting Information}

598 The Supporting Information is available free of charge at 599 https://pubs.acs.org/doi/10.1021/acsaem.9b02137.

600 Additional characterization data including TEM of PbSe 601 NCs after LD and FTIR, additional TE measurements, 602 and detailed explanation of the theoretical modeling of 603 the material TE properties (PDF)

\section{AUTHOR INFORMATION}

\section{Corresponding Authors}

606 *E-mail: dycadavidr@unal.edu.co.

607 *E-mail: acabot@irec.cat.

608 ORCID

609 Doris Cadavid: 0000-0002-1376-6078

610 Yu Liu: 0000-0001-7313-6740

611 Maria Ibáñez: 0000-0001-5013-2843

612 Joan Daniel Prades: 0000-0001-7055-5499

613 Andreu Cabot: 0000-0002-7533-3251

614 Notes

615 The authors declare no competing financial interest.

\section{$616 \square$ ACKNOWLEDGMENTS}

617 This work was supported by the Spanish Ministerio de 618 Economia y Competitividad through the project SEHTOP
(ENE2016-77798-C4-3-R) and the Generalitat de Catalunya 619 through the project 2017SGR1246. D.C. acknowledges support 620 from Universidad Nacional de Colombia. Y.L. acknowledges 621 funding from the European Union's Horizon 2020 research and 622 innovation programme under the Marie Sklodowska-Curie 623 grant agreement no. 754411. M.I. acknowledges financial 624 support from IST Austria.

625

\section{REFERENCES}

626

(1) Cadavid, D.; Ibáñez, M.; Gorsse, S.; López, A. M.; Cirera, A.; 627 Morante, J. R.; Cabot, A. Bottom-up Processing of Thermoelectric 628 Nanocomposites from Colloidal Nanocrystal Building Blocks: The 629 Case of Ag2Te-PbTe. J. Nanopart. Res. 2012, 14 (12), 1-10. 630

(2) Ibáñez, M.; Zamani, R.; Gorsse, S.; Fan, J.; Ortega, S.; Cadavid, D.; 631 Morante, J. R.; Arbiol, J.; Cabot, A. Core-Shell Nanoparticles As 632 Building Blocks for the Bottom-Up Production of Functional 633 Nanocomposites: PbTe-PbS Thermoelectric Properties. ACS Nano 634 2013, 7 (3), 2573-2586.

(3) Ortega, S.; Ibáñez, M.; Liu, Y.; Zhang, Y; Kovalenko, M. V.; 636 Cadavid, D.; Cabot, A. Bottom-up Engineering of Thermoelectric 637 Nanomaterials and Devices from Solution-Processed Nanoparticle 638 Building Blocks. Chem. Soc. Rev. 2017, 46 (12), 3510-3528.

(4) Ibáñez, M.; Luo, Z.; Genç, A.; Piveteau, L.; Ortega, S.; Cadavid, 640 D.; Dobrozhan, O.; Liu, Y.; Nachtegaal, M.; Zebarjadi, M.; et al. High- 641 Performance Thermoelectric Nanocomposites from Nanocrystal 642 Building Blocks. Nat. Commun. 2016, 7, 10766.

(5) Liu, Y.; Cadavid, D.; Ibáñez, M.; Ortega, S.; Martí-Sánchez, S.; 644 Dobrozhan, O.; Kovalenko, M. V.; Arbiol, J.; Cabot, A. Thermoelectric 645 Properties of Semiconductor-Metal Composites Produced by Particle 646 Blending. APL Mater. 2016, 4 (10), 104813.

(6) Liu, Y.; García, G.; Ortega, S.; Cadavid, D.; Palacios, P.; Lu, J.; 648 Ibáñez, M.; Xi, L.; Roo, J. D.; López, A. M.; et al. Solution-Based 649 Synthesis and Processing of $\mathrm{Sn}$ - and Bi-Doped Cu3SbSe4 Nanocrystals, 650 Nanomaterials and Ring-Shaped Thermoelectric Generators. J. Mater. 651 Chem. A 2017, 5 (6), 2592-2602. 652

(7) Liu, Y.; Zhang, Y.; Ortega, S.; Ibáñez, M.; Lim, K. H.; Grau- 653 Carbonell, A.; Martí-Sánchez, S.; Ng, K. M.; Arbiol, J.; Kovalenko, M. 654 V.; et al. Crystallographically Textured Nanomaterials Produced from 655 the Liquid Phase Sintering of BixSb2-XTe3 Nanocrystal Building 656 Blocks. Nano Lett. 2018, 18 (4), 2557-2563.

(8) Liu, Y.; Zhang, Y.; Lim, K. H.; Ibáñez, M.; Ortega, S.; Li, M.; 658 David, J.; Martí-Sánchez, S.; Ng, K. M.; Arbiol, J.; et al. High 659 Thermoelectric Performance in Crystallographically Textured N-Type 660 $\mathrm{Bi}_{2} \mathrm{Te}_{3-x} \mathrm{Se}_{x}$ Produced from Asymmetric Colloidal Nanocrystals. 661 ACS Nano 2018, 12 (7), 7174-7184.

(9) Zhang, Y.; Liu, Y.; Lim, K. H.; Xing, C.; Li, M.; Zhang, T.; Tang, P.; 663 Arbiol, J.; Llorca, J.; Ng, K. M.; et al. Tin Diselenide Molecular 664 Precursor for Solution-Processable Thermoelectric Materials. Angew. 665 Chem. 2018, 130 (52), 17309-17314.

(10) Li, M.; Liu, Y.; Zhang, Y.; Zuo, Y.; Li, J.; Lim, K. H.; Cadavid, D.; 667 Ng, K. M.; Cabot, A. Crystallographically Textured SnSe Nanomateri- 668 als Produced from the Liquid Phase Sintering of Nanocrystals. Dalton 669 Trans. 2019, 48 (11), 3641-3647.

(11) Lim, K. H.; Wong, K. W.; Liu, Y.; Zhang, Y.; Cadavid, D.; Cabot, 671 A.; Ng, K. M. Critical Role of Nanoinclusions in Silver Selenide 672 Nanocomposites as a Promising Room Temperature Thermoelectric 673 Material. J. Mater. Chem. C 2019, 7 (9), 2646-2652. 674

(12) Huynh, W. U.; Dittmer, J. J.; Alivisatos, A. P. Hybrid Nanorod- 675 Polymer Solar Cells. Science 2002, 295 (5564), 2425-2427. 676

(13) Kovalenko, M. V.; Scheele, M.; Talapin, D. V. Colloidal 677 Nanocrystals with Molecular Metal Chalcogenide Surface Ligands. 678 Science 2009, 324 (5933), 1417-1420.

(14) Kovalenko, M. V.; Bodnarchuk, M. I.; Zaumseil, J.; Lee, J.-S.; 680 Talapin, D. V. Expanding the Chemical Versatility of Colloidal 681 Nanocrystals Capped with Molecular Metal Chalcogenide Ligands. J. 682 Am. Chem. Soc. 2010, 132 (29), 10085-10092.

(15) Kovalenko, M. V.; Spokoyny, B.; Lee, J.-S.; Scheele, M.; Weber, 684 A.; Perera, S.; Landry, D.; Talapin, D. V. Semiconductor Nanocrystals 685 
686 Functionalized with Antimony Telluride Zintl Ions for Nanostructured 687 Thermoelectrics. J. Am. Chem. Soc. 2010, 132 (19), 6686-6695.

688 (16) Kovalenko, M. V.; Bodnarchuk, M. I.; Talapin, D. V. Nanocrystal 689 Superlattices with Thermally Degradable Hybrid Inorganic-Organic 690 Capping Ligands. J. Am. Chem. Soc. 2010, 132 (43), 15124-15126.

691 (17) Ibáñez, M.; Hasler, R.; Genç, A.; Liu, Y.; Kuster, B.; Schuster, M.; 692 Dobrozhan, O.; Cadavid, D.; Arbiol, J.; Cabot, A.; et al. Ligand693 Mediated Band Engineering in Bottom-Up Assembled SnTe Nano694 composites for Thermoelectric Energy Conversion. J. Am. Chem. Soc. 695 2019, 141 (20), 8025-8029.

696 (18) Dong, A.; Ye, X.; Chen, J.; Kang, Y.; Gordon, T.; Kikkawa, J. M.; 697 Murray, C. B. A Generalized Ligand-Exchange Strategy Enabling 698 Sequential Surface Functionalization of Colloidal Nanocrystals. J. Am. 699 Chem. Soc. 2011, 133 (4), 998-1006.

700 (19) Rosen, E. L.; Buonsanti, R.; Llordes, A.; Sawvel, A. M.; Milliron, 701 D. J.; Helms, B. A. Exceptionally Mild Reactive Stripping of Native 702 Ligands from Nanocrystal Surfaces by Using Meerwein's Salt. Angew. 703 Chem., Int. Ed. 2012, 51 (3), 684-689.

704 (20) Nag, A.; Kovalenko, M. V.; Lee, J.-S.; Liu, W.; Spokoyny, B.; 705 Talapin, D. V. Metal-Free Inorganic Ligands for Colloidal Nanocryst706 als: S2-, HS-, Se2-, $\mathrm{HSe}-, \mathrm{Te} 2-, \mathrm{HTe}-, \mathrm{TeS} 32-, \mathrm{OH}-$, and NH2707 as Surface Ligands. J. Am. Chem. Soc. 2011, 133 (27), 10612-10620. 708 (21) Chuang, C.-H. M.; Brown, P. R.; Bulović, V.; Bawendi, M. G. 709 Improved Performance and Stability in Quantum Dot Solar Cells 710 through Band Alignment Engineering. Nat. Mater. 2014, 13 (8), 796711801.

712 (22) Fan, J. Z.; Croix, A. D. L.; Yang, Z.; Howard, E.; Quintero713 Bermudez, R.; Levina, L.; Jenkinson, N. M.; Spear, N. J.; Li, Y.; 714 Ouellette, O.; et al. Ligand Cleavage Enables Formation of 1,2715 Ethanedithiol Capped Colloidal Quantum Dot Solids. Nanoscale 2019, 71611 (22), 10774-10781.

717 (23) Fafarman, A. T.; Koh, W.; Diroll, B. T.; Kim, D. K.; Ko, D.-K.; 718 Oh, S. J.; Ye, X.; Doan-Nguyen, V.; Crump, M. R.; Reifsnyder, D. C.; 719 et al. Thiocyanate-Capped Nanocrystal Colloids: Vibrational Reporter 720 of Surface Chemistry and Solution-Based Route to Enhanced Coupling 721 in Nanocrystal Solids. J. Am. Chem. Soc. 2011, 133 (39), 15753-15761. 722 (24) Koh, W.; Saudari, S. R.; Fafarman, A. T.; Kagan, C. R.; Murray, C. 723 B. Thiocyanate-Capped PbS Nanocubes: Ambipolar Transport Enables 724 Quantum Dot Based Circuits on a Flexible Substrate. Nano Lett. 2011, 72511 (11), 4764-4767.

726 (25) Zhang, H.; Hu, B.; Sun, L.; Hovden, R.; Wise, F. W.; Muller, D. 727 A.; Robinson, R. D. Surfactant Ligand Removal and Rational 728 Fabrication of Inorganically Connected Quantum Dots. Nano Lett. 7292011,11 (12), 5356-5361.

730 (26) Nag, A.; Chung, D. S.; Dolzhnikov, D. S.; Dimitrijevic, N. M.; 731 Chattopadhyay, S.; Shibata, T.; Talapin, D. V. Effect of Metal Ions on 732 Photoluminescence, Charge Transport, Magnetic and Catalytic 733 Properties of All-Inorganic Colloidal Nanocrystals and Nanocrystal 734 Solids. J. Am. Chem. Soc. 2012, 134 (33), 13604-13615.

735 (27) Ibáñez, M.; Hasler, R.; Liu, Y.; Dobrozhan, O.; Nazarenko, O.; 736 Cadavid, D.; Cabot, A.; Kovalenko, M. V. Tuning P-Type Transport in 737 Bottom-Up-Engineered Nanocrystalline $\mathrm{Pb}$ Chalcogenides Using 738 Alkali Metal Chalcogenides as Capping Ligands. Chem. Mater. 2017, 73929 (17), 7093-7097.

740 (28) Ibáñez, M.; Genç, A.; Hasler, R.; Liu, Y.; Dobrozhan, O.; 741 Nazarenko, O.; Mata, M. de la; Arbiol, J.; Cabot, A.; Kovalenko, M. V. 742 Tuning Transport Properties in Thermoelectric Nanocomposites 743 through Inorganic Ligands and Heterostructured Building Blocks. 744 ACS Nano 2019, 13 (6), 6572-6580.

745 (29) Ibáñez, M.; Korkosz, R. J.; Luo, Z.; Riba, P.; Cadavid, D.; Ortega, 746 S.; Cabot, A.; Kanatzidis, M. G. Electron Doping in Bottom-Up 747 Engineered Thermoelectric Nanomaterials through HCl-Mediated 748 Ligand Displacement. J. Am. Chem. Soc. 2015, 137 (12), 4046-4049. 749 (30) Tang, J.; Kemp, K. W.; Hoogland, S.; Jeong, K. S.; Liu, H.; Levina, 750 L.; Furukawa, M.; Wang, X.; Debnath, R.; Cha, D.; et al. Colloidal751 Quantum-Dot Photovoltaics Using Atomic-Ligand Passivation. Nat. 752 Mater. 2011, 10 (10), 765-771.
(31) Zhitomirsky, D.; Furukawa, M.; Tang, J.; Stadler, P.; Hoogland, 753 S.; Voznyy, O.; Liu, H.; Sargent, E. H. N-Type Colloidal-Quantum-Dot 754 Solids for Photovoltaics. Adv. Mater. 2012, 24 (46), 6181-6185. 755 (32) Ning, Z.; Ren, Y.; Hoogland, S.; Voznyy, O.; Levina, L.; Stadler, 756 P.; Lan, X.; Zhitomirsky, D.; Sargent, E. H. All-Inorganic Colloidal 757 Quantum Dot Photovoltaics Employing Solution-Phase Halide 758 Passivation. Adv. Mater. 2012, 24 (47), 6295-6299.

(33) Nag, A.; Zhang, H.; Janke, E.; Talapin, D. V. Inorganic Surface 760 Ligands for Colloidal Nanomaterials. Z. Phys. Chem. 2015, 229 (1-2), 761 85-107.

762

(34) Anderson, N. C.; Owen, J. S. Soluble, Chloride-Terminated 763 CdSe Nanocrystals: Ligand Exchange Monitored by $1 \mathrm{H}$ and 31P NMR 764 Spectroscopy. Chem. Mater. 2013, 25 (1), 69-76.

(35) Jung, J.; Chang, M.; Yoon, H. Interface Engineering Strategies for 766 Fabricating Nanocrystal-Based Organic-Inorganic Nanocomposites. 767 Appl. Sci. 2018, 8 (8), 1376.

(36) Hewavitharana, I. K.; Brock, S. L. When Ligand Exchange Leads 769 to Ion Exchange: Nanocrystal Facets Dictate the Outcome. ACS Nano 770 2017, 11 (11), 11217-11224.

771

(37) Cadavid, D.; Ibáñez, M.; Shavel, A.; Durá, O. J.; de la Torre, M. A. 772 L.; Cabot, A. Organic Ligand Displacement by Metal Salts to Enhance 773 Nanoparticle Functionality: Thermoelectric Properties of Ag2Te. J. 774 Mater. Chem. A 2013, 1 (15), 4864-4870.

(38) Ibáñez, M.; Cadavid, D.; Zamani, R.; García-Castelló, N.; 776 Izquierdo-Roca, V.; Li, W.; Fairbrother, A.; Prades, J. D.; Shavel, A.; 777 Arbiol, J.; et al. Composition Control and Thermoelectric Properties of 778 Quaternary Chalcogenide Nanocrystals: The Case of Stannite 779 Cu2CdSnSe4. Chem. Mater. 2012, 24 (3), 562-570.

780

(39) Ibáñez, M.; Zamani, R.; LaLonde, A.; Cadavid, D.; Li, W.; Shavel, 781 A.; Arbiol, J.; Morante, J. R.; Gorsse, S.; Snyder, G. J.; et al. 782 Cu2ZnGeSe4 Nanocrystals: Synthesis and Thermoelectric Properties. 783 J. Am. Chem. Soc. 2012, 134 (9), 4060-4063.

784

(40) Ibáñez, M.; Zamani, R.; Li, W.; Shavel, A.; Arbiol, J.; Morante, J. 785 R.; Cabot, A. Extending the Nanocrystal Synthesis Control to 786 Quaternary Compositions. Cryst. Growth Des. 2012, 12 (3), 1085- 787 1090.

788

(41) Shavel, A.; Cadavid, D.; Ibáñez, M.; Carrete, A.; Cabot, A. 789 Continuous Production of Cu2ZnSnS4 Nanocrystals in a Flow Reactor. 790 J. Am. Chem. Soc. 2012, 134 (3), 1438-1441.

(42) Ibáñez, M.; Zamani, R.; Li, W.; Cadavid, D.; Gorsse, S.; Katcho, 792 N. A.; Shavel, A.; López, A. M.; Morante, J. R.; Arbiol, J. 793 Crystallographic Control at the Nanoscale to Enhance Functionality: 794 Polytypic Cu2GeSe3 Nanoparticles as Thermoelectric Materials. Chem. 795 Mater. 2012, 24, 4615.

(43) Ibáñez, M.; Cadavid, D.; Tamburini, U. A.; Zamani, R.; Gorsse, 797 S.; Li, W.; Shavel, A.; López, A. M.; Morante, J. R.; Arbiol, J. Colloidal 798 Synthesis and Thermoelectric Properties of Cu2SnSe3 Nanocrystals. J. 799 Mater. Chem. A 2013, 1, 1421.

800

(44) Martin, J.; Wang, L.; Chen, L.; Nolas, G. S. Enhanced Seebeck 801 Coefficient through Energy-Barrier Scattering in PbTe Nanocompo- 802 sites. Phys. Rev. B: Condens. Matter Mater. Phys. 2009, 79 (11), 115311. 803

(45) Mentzel, T. S.; Porter, V. J.; Geyer, S.; MacLean, K.; Bawendi, M. 804 G.; Kastner, M. A. Charge Transport in PbSe Nanocrystal Arrays. Phys. 805 Rev. B: Condens. Matter Mater. Phys. 2008, 77 (7), No. 075316.

(46) Ziman, J. M. Principles of the Theory of Solids; University Press: 807 Cambridge, U.K., 1972.

(47) Zhao, L.-D.; Tan, G.; Hao, S.; He, J.; Pei, Y.; Chi, H.; Wang, H.; 809 Gong, S.; Xu, H.; Dravid, V. P.; et al. Ultrahigh Power Factor and 810 Thermoelectric Performance in Hole-Doped Single-Crystal SnSe. 811 Science 2016, 351 (6269), 141-144.

(48) Cui, H.; Liu, H.; Li, X.; Wang, J.; Han, F.; Zhang, X.; Boughton, 813 R. I. Synthesis of Bi2Se3 Thermoelectric Nanosheets and Nanotubes 814 through Hydrothermal Co-Reduction Method. J. Solid State Chem. 815 2004, 177 (11), 4001-4006.

816

(49) Gautam, U. K.; Rajamathi, M.; Meldrum, F.; Morgan, P.; 817 Seshadri, R. A Solvothermal Route to Capped CdSe Nanoparticles. 818 Chem. Commun. 2001, No. 7, 629-630.

(50) Qu, B.; Zhang, M.; Lei, D.; Zeng, Y.; Chen, Y.; Chen, L.; Li, Q.; 820 Wang, Y.; Wang, T. Facile Solvothermal Synthesis of Mesoporous 821 
$822 \mathrm{Cu} 2 \mathrm{SnS} 3$ Spheres and Their Application in Lithium-Ion Batteries. 823 Nanoscale 2011, 3 (9), 3646-3651.

824 (51) Cui, H.; Liu, H.; Wang, J.; Li, X.; Han, F.; Boughton, R. I. 825 Sonochemical Synthesis of Bismuth Selenide Nanobelts at Room 826 Temperature. J. Cryst. Growth 2004, 271 (3), 456-461.

827 (52) Harpeness, R.; Palchik, O.; Gedanken, A.; Palchik, V.; Amiel, S.; 828 Slifkin, M. A.; Weiss, A. M. Preparation and Characterization of Ag2E $829(\mathrm{E}=\mathrm{Se}, \mathrm{Te})$ Using the Sonochemically Assisted Polyol Method. Chem. 830 Mater. 2002, 14 (5), 2094-2102.

831 (53) Arachchige, I. U.; Brock, S. L. Sol-Gel Methods for the Assembly 832 of Metal Chalcogenide Quantum Dots. Acc. Chem. Res. 2007, 40 (9), 833 801-809.

834 (54) Min, Y.; Roh, J. W.; Yang, H.; Park, M.; Kim, S. I.; Hwang, S.; Lee, 835 S. M.; Lee, K. H.; Jeong, U. Surfactant-Free Scalable Synthesis of $836 \mathrm{Bi} 2 \mathrm{Te} 3$ and Bi2Se3 Nanoflakes and Enhanced Thermoelectric 837 Properties of Their Nanocomposites (Adv. Mater. 10/2013). Adv. 838 Mater. 2013, 25 (10), 1424-1424.

839 (55) Liu, H.; Cui, H.; Han, F.; Li, X.; Wang, J.; Boughton, R. I. Growth 840 of Bi2Se3 Nanobelts Synthesized through a Co-Reduction Method 841 under Ultrasonic Irradiation at Room Temperature. Cryst. Growth Des. 842 2005, 5 (5), 1711-1714.

843 (56) Ibáñez, M.; Guardia, P.; Shavel, A.; Cadavid, D.; Arbiol, J.; 844 Morante, J. R.; Cabot, A. Growth Kinetics of Asymmetric Bi2S3 845 Nanocrystals: Size Distribution Focusing in Nanorods. J. Phys. Chem. C 8462011,115 (16), 7947-7955.

847 (57) Parker, D.; Singh, D. J. High-Temperature Thermoelectric 848 Performance of Heavily Doped PbSe. Phys. Rev. B: Condens. Matter 849 Mater. Phys. 2010, 82 (3), No. 035204.

850 (58) Zhao, L.-D.; Hao, S.; Lo, S.-H.; Wu, C.-I.; Zhou, X.; Lee, Y.; Li, 851 H.; Biswas, K.; Hogan, T. P.; Uher, C.; et al. High Thermoelectric 852 Performance via Hierarchical Compositionally Alloyed Nanostruc853 tures. J. Am. Chem. Soc. 2013, 135 (19), 7364-7370.

854 (59) Zhou, C.; Lee, Y. K.; Cha, J.; Yoo, B.; Cho, S.-P.; Hyeon, T.; 855 Chung, I. Defect Engineering for High-Performance n-Type PbSe 856 Thermoelectrics. J. Am. Chem. Soc. 2018, 140 (29), 9282-9290.

857 (60) Huang, Z.; Zhang, Y.; Wu, H.; Pennycook, S. J.; Zhao, L.-D. 858 Enhancing Thermoelectric Performance of P-Type PbSe through 859 Suppressing Electronic Thermal Transports. ACS Appl. Energy Mater. 860 2019, 2 (11), 8236-8243.

861 (61) Slade, T. J.; Bailey, T. P.; Grovogui, J. A.; Hua, X.; Zhang, X.; 862 Kuo, J. J.; Hadar, I.; Snyder, G. J.; Wolverton, C.; Dravid, V. P.; et al. 863 High Thermoelectric Performance in $\mathrm{PbSe}-\mathrm{NaSbSe} 2$ Alloys from 864 Valence Band Convergence and Low Thermal Conductivity. Adv. 865 Energy Mater. 2019, 9 (30), 1901377.

866 (62) Urban, J. J.; Talapin, D. V.; Shevchenko, E. V.; Murray, C. B. Self867 Assembly of $\mathrm{PbTe}$ Quantum Dots into Nanocrystal Superlattices and 868 Glassy Films. J. Am. Chem. Soc. 2006, 128 (10), 3248-3255.

869 (63) Wang, H.; Pei, Y.; LaLonde, A. D.; Snyder, G. J. Heavily Doped 870 P-Type PbSe with High Thermoelectric Performance: An Alternative 871 for PbTe. Adv. Mater. 2011, 23 (11), 1366-1370.

872 (64) Ravich, Yu. I.; Efimova, B. A.; Tamarchenko, V. I. Semiconducting 873 Lead Chalcogenides; Plenum: New York, 1970.

874 (65) Androulakis, J.; Chung, D.-Y.; Su, X.; Zhang, L.; Uher, C.; 875 Hasapis, T. C.; Hatzikraniotis, E.; Paraskevopoulos, K. M.; Kanatzidis, 876 M. G. High-Temperature Charge and Thermal Transport Properties of 877 the n-Type Thermoelectric Material PbSe. Phys. Rev. B: Condens. Matter 878 Mater. Phys. 2011, 84 (15), 155207.

879 (66) Scheele, M.; Oeschler, N.; Veremchuk, I.; Peters, S.-O.; Littig, A.; 880 Kornowski, A.; Klinke, C.; Weller, H. Thermoelectric Properties of 881 Lead Chalcogenide Core-Shell Nanostructures. ACS Nano 2011, 5 882 (11), 8541-8551.

883 (67) Mokurala, K.; Mallick, S. Effect of Annealing Atmosphere on 884 Quaternary Chalcogenide-Based Counter Electrodes in Dye-Sensitized 885 Solar Cell Performance: Synthesis of $\mathrm{Cu} 2 \mathrm{FeSnS4} 4$ and $\mathrm{Cu} 2 \mathrm{CdSnS} 4$ 886 Nanoparticles by Thermal Decomposition Process. RSC Adv. 2017, 7 887 (25), 15139-15148.

888 (68) Bhattacharya, S.; Pal, A.; Jana, A.; Datta, J. Synthesis and 889 Characterization of CdS Nanoparticles Decorated TiO2Matrix for an
Efficient N3 Based Dye Sensitized Solar Cell (DSSC). J. Mater. Sci.: 890 Mater. Electron. 2016, 27 (12), 12438-12445.

(69) Mori, T. Novel Principles and Nanostructuring Methods for 892 Enhanced Thermoelectrics. Small 2017, 13 (45), 1702013.

(70) Zheng, X.; Hou, Y.; Sun, H.-T.; Mohammed, O. F.; Sargent, E. 894 H.; Bakr, O. M. Reducing Defects in Halide Perovskite Nanocrystals for 895 Light-Emitting Applications. J. Phys. Chem. Lett. 2019, 10 (10), 2629- 896 2640.

(71) Carey, G. H.; Abdelhady, A. L.; Ning, Z.; Thon, S. M.; Bakr, O. 898 M.; Sargent, E. H. Colloidal Quantum Dot Solar Cells. Chem. Rev. 2015, 899 115 (23), 12732-12763.

(72) Wang, S.; Zheng, G.; Luo, T.; She, X.; Li, H.; Tang, X. Exploring 901 the Doping Effects of Ag in P-Type PbSe Compounds with Enhanced 902 Thermoelectric Performance. J. Phys. D: Appl. Phys. 2011, 44 (47), 903 475304.

904

(73) Zhang, Q.; Wang, H.; Liu, W.; Wang, H.; Yu, B.; Zhang, Q.; Tian, 905 Z.; Ni, G.; Lee, S.; Esfarjani, K.; et al. Enhancement of Thermoelectric 906 Figure-of-Merit by Resonant States of Aluminium Doping in Lead 907 Selenide. Energy Environ. Sci. 2012, 5 (1), 5246-5251. 908

(74) Shanker, G. S.; Swarnkar, A.; Chatterjee, A.; Chakraborty, S.; 909 Phukan, M.; Parveen, N.; Biswas, K.; Nag, A. Electronic Grade and 910 Flexible Semiconductor Film Employing Oriented Attachment of 911 Colloidal Ligand-Free PbS and PbSe Nanocrystals at Room Temper- 912 ature. Nanoscale 2015, 7 (20), 9204-9214.

(75) Jin, R.; Chen, G.; Pei, J.; Sun, J.; Wang, Q. Controllable Synthesis 914 and Thermoelectric Transport Properties of Binary-Phased PbTe/ 915 PbSe Nanocrystals. CrystEngComm 2012, 14 (13), 4461-4466. 916

(76) Marks, B. M.; Howard, H. C. The Catalytic Decomposition of 917 Oleic Acid. J. Phys. Chem. 1928, 32 (7), 1040-1048.

(77) Rowe, D. M. Thermoelectrics Handbook: Macro to Nano; CRC 919 Press INC: 2006.

(78) Goupil, C.; Seifert, W.; Zabrocki, K.; Müller, E.; Snyder, G. J. 921 Thermodynamics of Thermoelectric Phenomena and Applications. 922 Entropy 2011, 13 (8), 1481-1517.

(79) Min, G.; Rowe, D. M. Ring-Structured Thermoelectric Module. 924 Semicond. Sci. Technol. 2007, 22 (8), 880-883. 